\title{
Mobile Three Gas Extractor Using Pressure Swing Adsorption Method
}

\author{
Pascalin Tiam Kapen ${ }^{1,2}$, Tendong Tadadjeu Neville ${ }^{1}$, Dongmeza Koudjou Jauspin ${ }^{1}$ \\ ${ }^{1}$ Université des Montagnes, ISST, Bangangté-Cameroon \\ ${ }^{2}$ University of Dschang, IUT-FV, LISIE/L2MSP, Bandjoun-Cameroon \\ fpascalin20022003@gmail.com; tadadjeuneville@yahoo.fr; dkjauspin@gmail.com
}

\begin{abstract}
This paper deals with a simple approach of producing three gases that are oxygen, nitrogen and pressurized air by using a mobile three gas extractor. Indeed, the proposed medical device integrates the following modules driven by an Arduino Mega 2560 board: Module of filtration and production of air made of filters, compressor and a cooling coil; module of oxygen and nitrogen production based on pressure swing adsorption (PSA) method and using the zeolite molecular sieves that restrain nitrogen and produce oxygen. The device is equipped of pressure sensors to control the output pressure of the gases. This implemented equipment has been tested and we obtained promising results. Indeed, a percentage of oxygen of $82 \%$ has been reached. We have produced pressurized air with a pressure of 2.5 bars.
\end{abstract}

Key words: Mobile Tree Gas Extractor; Oxygen; Nitrogen; Pressurized Air; Pressure Swing Adsorption (PSA).

\section{Introduction}

The supply of oxygen, nitrogen and pressurized air is a major problem in hospitals in developing countries [1, 2]. The usefulness of these gases is no longer to demonstrate. Indeed, oxygen is used for patients suffering from hypoxia; nitrogen is used for the conservation of physiological fluids like blood and many devices work with pressurized air. It is therefore interesting to start thinking about a simple equipment capable of producing such gases. It is in this vein, that we propose a simple mobile three gas extractor using pressure swing adsorption method.

\section{Material and methods}

\subsection{Material}

The electronic components used in our work are made of:

- Two filters, for the elimination of air particles having a diameter more than 2.5 micro-meters or less than 2.5 micro-meters;

- A compressor used for bringing up the pressure of air at 3 bars; 
- A cooling coil used for cooling the pressurized air;

- Two molecular sieves used to trap nitrogen and produce oxygen;

- A PT100 temperature sensor, used for measuring the temperature of oxygen;

- Three HK 3023 pressure sensors, for measuring the pressure of various gases;

- A pressure regulator, used in regulating the pressure of the compressed air, that is 3 bars, to the atmospheric pressure that is 1.01325 bar;

- An oxygen sensor, used in measuring the concentration of oxygen;

- A flow meter, used in regulating the flow of oxygen;

- A humidifier, used in humidifying oxygen given to the patient;

- An ATMEgA 2560 microcontroller card for commanding the entire system;

- Valves, used for commanding air, nitrogen and oxygen circulation.

The operating diagram of our equipment is shown in figure 1 :

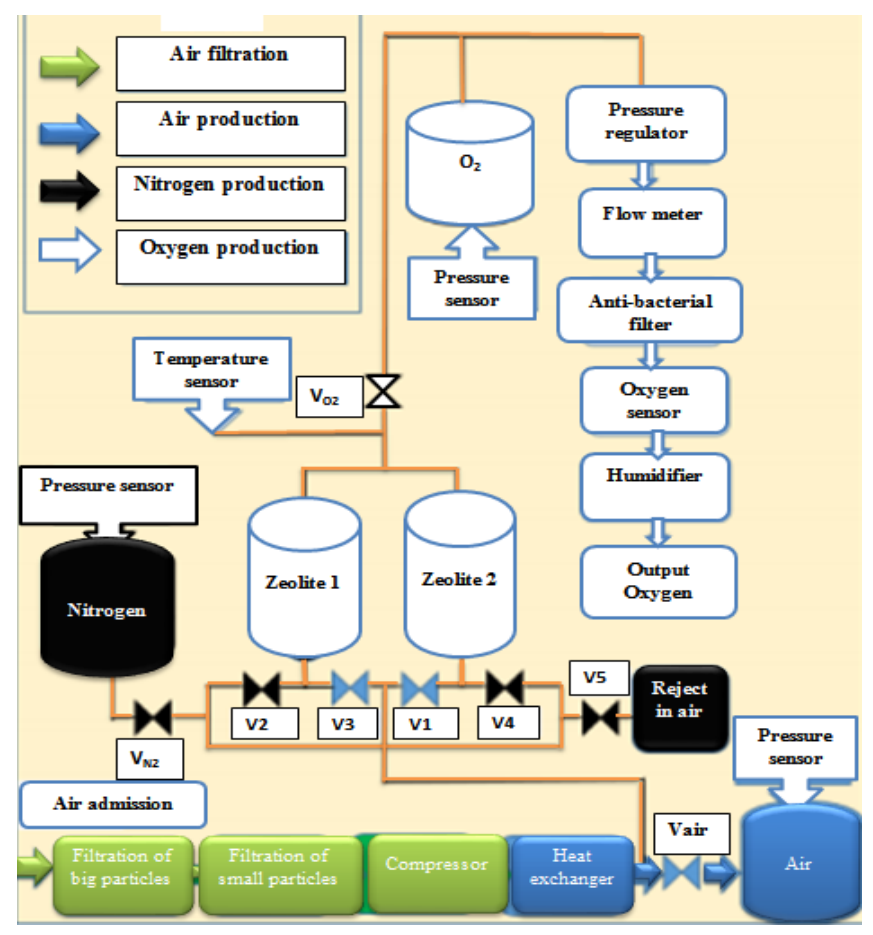

Figure 1 : Operating diagram

Where

$\mathrm{V}_{\mathrm{N} 2}, \mathrm{~V}_{2}$ and $\mathrm{V}_{4}$ are the electrical valves admitting the flow of Nitrogen;

$V_{02}, V_{3}$ and $V_{1}$ are the electrical valves admitting the flow of Oxygen;

$V_{\text {air }}$ is the electrical valve admitting the flow of pressurized air. 
The Arduino Mega 2560 board driving the different modules is presented in figure 2.

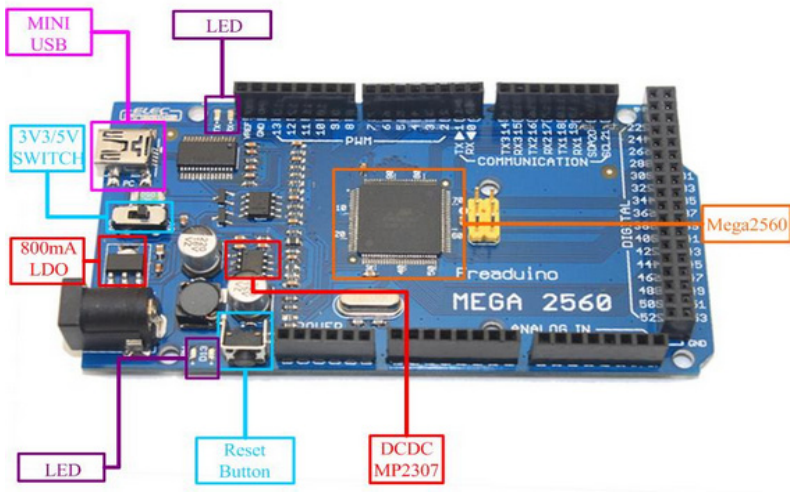

Figure 2 : Arduino Mega 2560 board

The electronic card incorporating the Arduino Mega 2560 board and developed in this work can be visualized in figure 3.

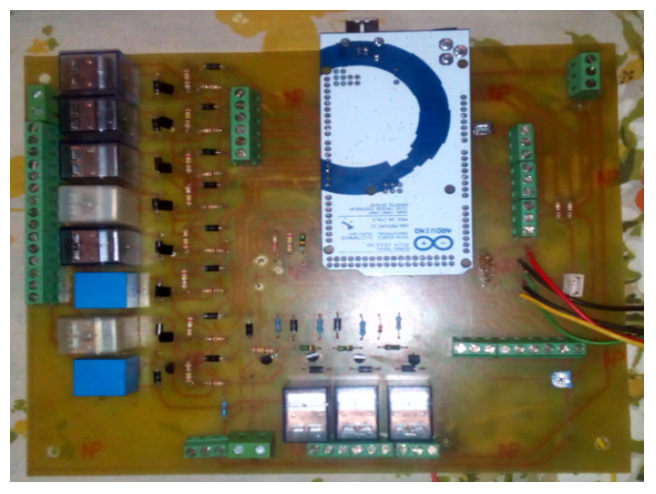

Figure 3: Electronic card commanding all the modules

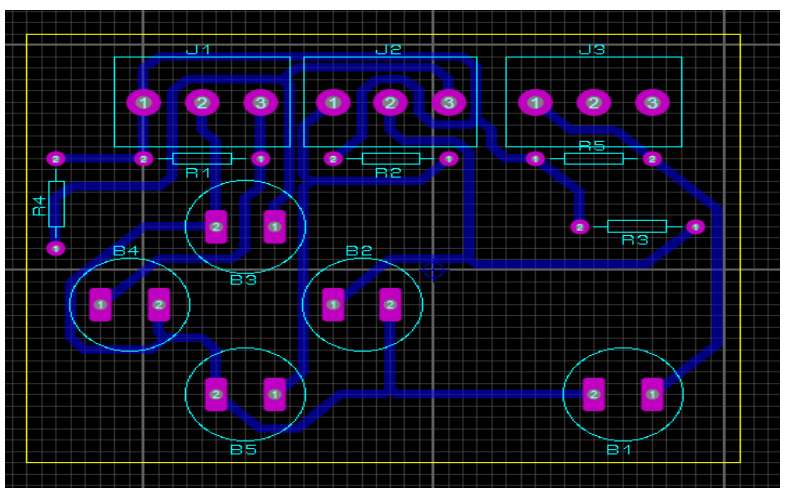

Figure 4: ARES circuit of the electronic card commanding all the modules

\subsection{Methods}

\subsubsection{Module of filtration and production of pressurized air}

The filtration of air is provided by two filters, one for restraining particles of more than 2.5 micro-meters of diameter and the other one used for restraining particles of less than 2.5 micro-meters of diameter. A compressor is used for elevating the pressure of air till 3 bars. Then a cooling coil is used for cooling the air till the atmospheric temperature. We use a pressure sensor to control the pressure of air.

\subsubsection{Module of production of oxygen}

In order to produce oxygen, the pressure swing adsorption (PSA) method has been implemented. Indeed, the approach consists of the production of oxygen passes through the usage of two zeolite molecular sieves. These sieves are used to restrain nitrogen molecules and produce oxygen. A PT100 temperature sensor has been used to measure the temperature of oxygen produced, in order to secure the patient. In addition, a pressure regulator has been used for regulating the pressure of air from the value of 3 bars to the value of 1.1325 bar. An oxygen sensor has been incorporated for to measure of 
oxygen concentration. A flow meter is used for regulating the flow of oxygen and a humidifier for humidifying the gas administrated to the patient.

\subsubsection{Module of production of nitrogen}

By injecting the oxygen at the outlet of zeolite molecular sieves, nitrogen restrained by the zeolite is liberated and can be stored in a tank.

\subsubsection{Module of display on an LCD screen $20 * 4$}

We used an LCD screen $20 * 4$. The information displayed is:

- Oxygen temperature;

- Oxygen pressure;

- Pressurized air pressure.

\section{Results and discussion}

Our equipment was tested and we obtained promising results.

\subsection{Module of filtration and production of pressurized air}

We made a test by using a HK 3023 pressure sensor in order to control the pressure of the pressurized air at the outlet of our circuit. The result is shown is figure 5 .

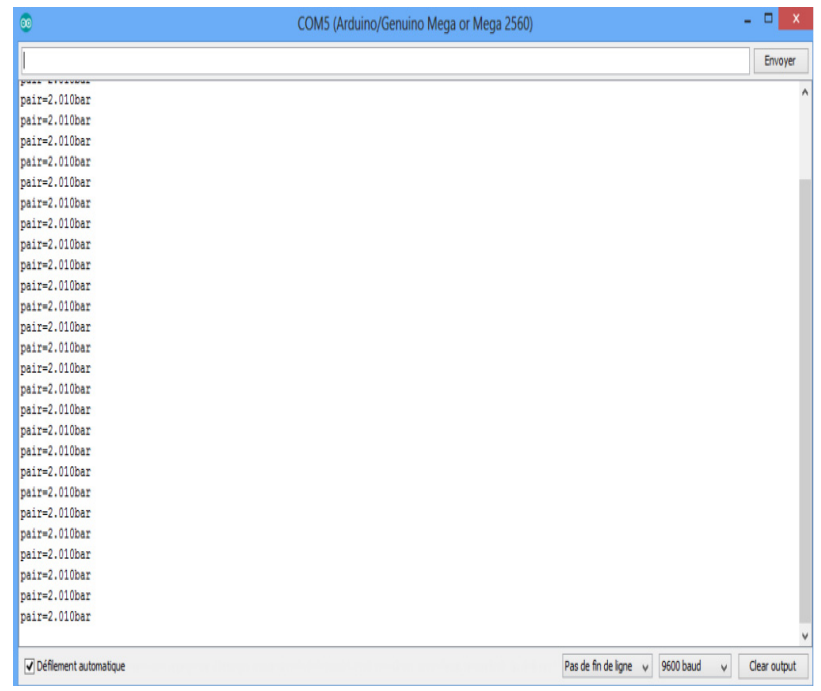

Figure 5: Screenshot showing the pressurized air pressure evolution from the serial monitor of the Arduino Mega 2560 board

\subsection{Module of production of oxygen}

The oxygen temperature and pressure are visualized from a serial monitor of the Arduino Mega 2560 board by using a PT100 temperature sensor and a HK 3023 pressure sensor. The results are presented in figures 6 and 7. 


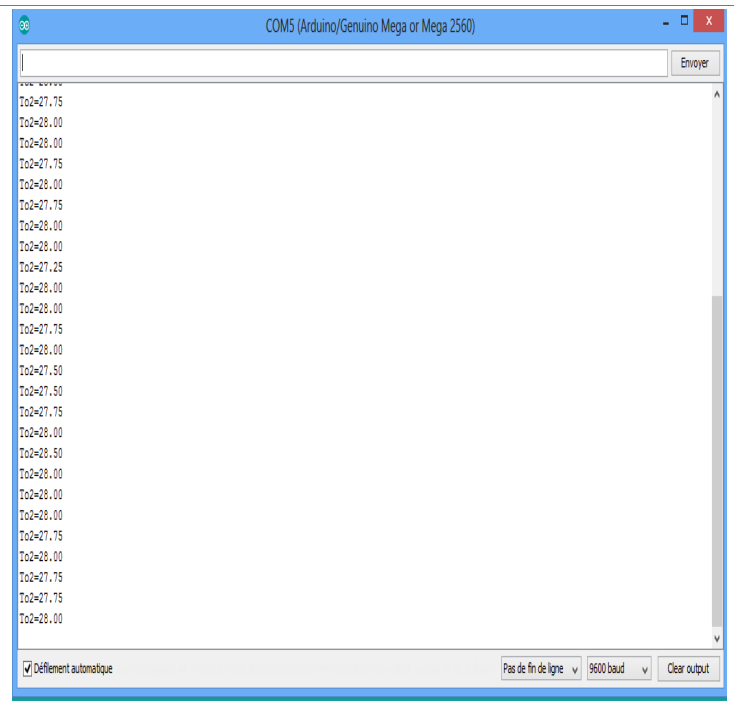

Figure 6: Screenshot showing the oxygen temperature evolution from the serial monitor of the Arduino Mega 2560 board

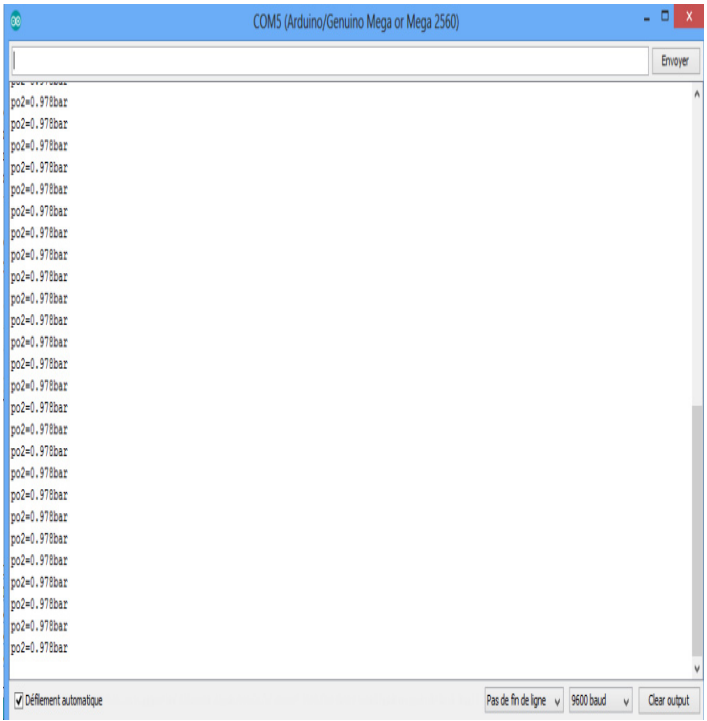

Figure 7: Screenshot showing the oxygen pressure evolution from the serial monitor of the Arduino Mega 2560 board

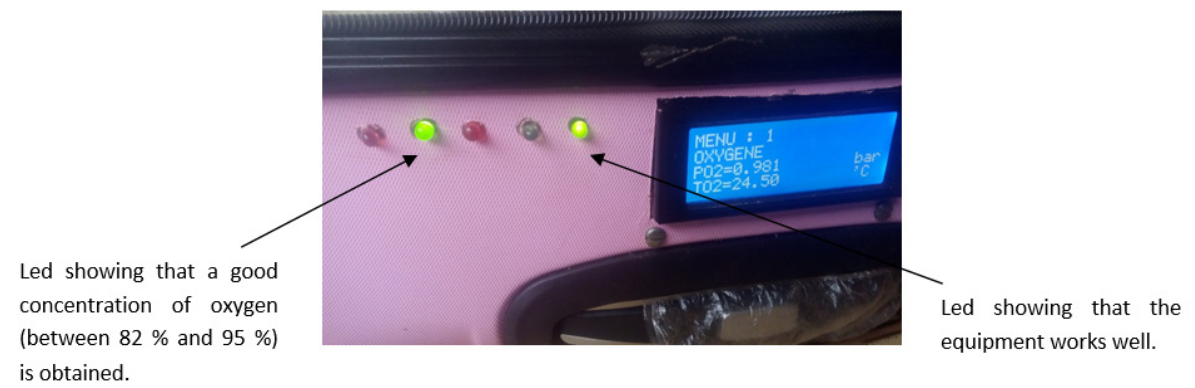

Figure 8: Test of oxygen concentration

An oxygen concentration (between $82 \%$ and $95 \%$ ) obtained with the equipment is shown on the LCD screen in figure 8. 
The final equipment developed in this work is presented in figure 9.
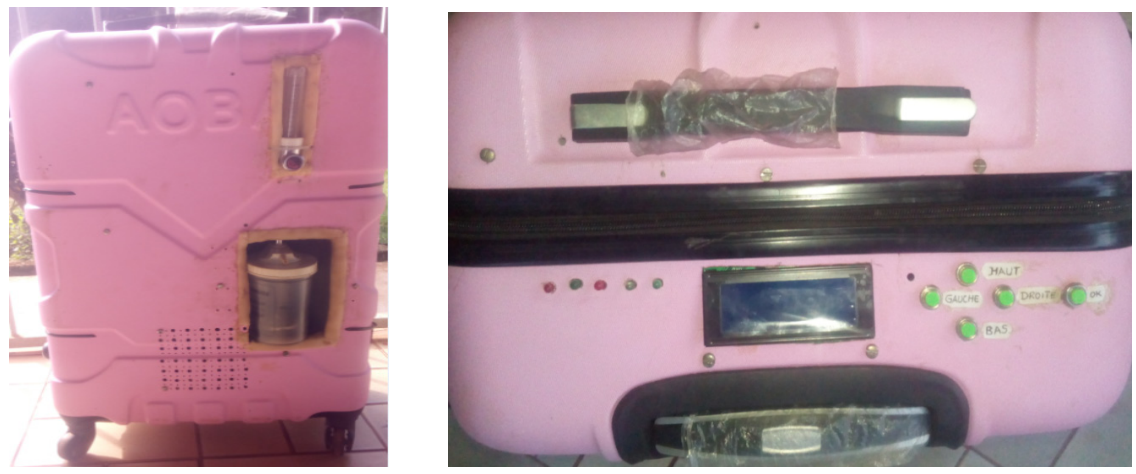

Figure 9: Different views of the final equipment

\section{Conclusion}

In this paper, a mobile three gas extractor using pressure swing adsorption method has been developed. Indeed, the equipment developed consisted of a module of filtration and production of air made of filters, compressor and a cooling coil; a module of oxygen and nitrogen production based on pressure swing adsorption (PSA) method and using the zeolite molecular sieves that restrain nitrogen and produce oxygen. It can be concluded that the implemented medical device produced a good percentage of oxygen between $82 \%$ and $95 \%$. In addition, a pressure of 2.5 bars has been reached for the pressurized air. It would be useful to improve the equipment by adding a hybrid enery supplier in case of electrical power failure. It would be also important to reinforce a security system. All this constitutes the ways of investigation of future works.

\section{REFERENCES}

[1] Mullie, A. (2010). Les gaz à usage médical, des produits thérapeutiques pas comme les autres: aspects spécifiques de l'application de la législation pharmaceutique, de son enregistrement à sa distribution (Doctoral dissertation).

[2] Organisation mondiale de la Santé (2016). Spécifications techniques pour les concentrateurs d'oxygène. 